\title{
MODULATION-MODE ASSIGNMENT FOR SVD-ASSISTED MULTIUSER MIMO SYSTEMS WITH CORRELATION
}

\author{
César Benavente-Peces, Francisco Cano-Broncano \\ Universidad Politécnica de Madrid, Ctra. Valencia. km. 7, 28031 Madrid, Spain \\ cesar.benavente@upm.es \\ Sebastian Aust, Andreas Ahrens \\ Hochschule Wismar, University of Technology, Business and Design \\ Philipp-Müller-Straße 14, 23966 Wismar, Germany \\ andreas.ahrens@hs-wismar.de
}

\begin{abstract}
Keywords: Multiple-Input Multiple-Output System, Singular-Value Decomposition, Bit Allocation, Power Allocation, Wireless Transmission, Multiuser Transmission.

Abstract: $\quad$ Multiuser multiple-input multiple-output (MIMO) downlink (DL) transmission schemes experience both multiuser interference as well as inter-antenna interference. The singular value decomposition provides an appropriate mean to process channel information and allows us to take the individual user's channel characteristics into account rather than treating all users channels jointly as in zero-forcing (ZF) multiuser transmission techniques. However, uncorrelated MIMO channels has attracted a lot of attention and reached a state of maturity. By contrast, the performance analysis in the presence of antenna fading correlation, which decreases the channel capacity, requires substantial further research. The joint optimization of the number of activated MIMO layers and the number of bits per symbol along with the appropriate allocation of the transmit power shows that not necessarily all user-specific MIMO layers has to be activated in order to minimize the overall BER under the constraint of a given fixed data throughput.
\end{abstract}

\section{INTRODUCTION}

Adaptive modulation is a promising technique to increase the spectral efficiency of wireless communication systems by adapting the signal parameters, such as modulation constellation or transmit power, dynamically to changing channel conditions. However, in order to comply with the demand on increasing available data rates in particular in wireless technologies, systems with multiple transmit and receive antennas, also called MIMO systems, have become indispensable and can be considered as an essential part of increasing both the achievable capacity and integrity of future generations of wireless systems (Kühn, 2006). Besides, single-user MIMO transmission schemes for both non-frequency and frequency selective MIMO channels have attracted a lot of attention and reached a state of maturity (Kühn, 2006; Ahrens and Lange, 2008). By contrast, MIMO-aided multiple-user systems require substantial further research where both multiuser as well as multi-antenna interferences have to be taken into account.

Considering the entirety of the antennas of all mo- bile terminals at one end and the antennas of the base station at the other end of the communication link, state of the art interference cancellation is based on a central signal processing unit, e.g. a central unit at the base station, where joint detection can be applied in the uplink (UL) and joint transmission in the downlink (DL), respectively (Meurer et al., 2000; Choi and Murch, 2004; Joham et al., 2005). Widely used linear preprocessing techniques such as Minimum Mean Square Error or Zero Forcing (ZF) have attracted a lot of research and have reached a state of maturity too (Choi and Murch, 2003). Therefore, in this work SVD-assisted downlink (DL) multiuser multiple-input multiple-output (MIMO) systems are considered, which take the individual user's channel characteristics into account (Ahrens and BenaventePeces, 2010; Liu et al., 2008) rather than treating all users channels jointly as in ZF multiuser transmission techniques. Treating all user independently, adaptive modulation is a promising technique to increase the spectral efficiency of wireless transmission systems by adapting the signal parameters, such as modulation constellation or transmit power, dynamically 
to individually changing channel conditions, where the most beneficial choice of the number of activated user-specific MIMO layers together with the number of bits per symbol and the appropriate allocation of the available transmit power offer a certain degree of design freedom, which substantially affects the performance of MIMO systems.

Against this background, in this paper a SVDassisted multiuser MIMO scheme is investigated, where both multiuser interferences as well as multiantenna interferences are perfectly eliminated. The novel contribution of this paper is that we demonstrate the benefits of amalgamating a suitable choice of activated MIMO layers and number of bits per symbol along with the appropriate allocation of the transmit power under the constraint of a given fixed data throughput. Besides the signal processing needed to perfectly separate the users within a multiuser system, multiple antenna systems are affected by signal correlation among antennas, which produces a degradation of the link performance, depending on the physical relative position of the antennas and the mobility of the front-ends and has to be taken into consideration.

The remaining part of this paper is organized as follows: Section 2 introduces the multiuser system model, while the considered quality criteria are briefly reviewed in section 3 . The associated performance results are presented and interpreted in section 4 . Finally, section 5 provides some concluding remarks.

\section{MULTIUSER SYSTEM MODEL}

The system model considered in this work consists of a single base station (BS) supporting $K$ mobile stations (MSs). The BS is equipped with $n_{\mathrm{T}}$ transmit antennas, while the $k$ th (with $k=1, \ldots, K$ ) MS has $n_{\mathrm{R} k}$ receive antennas, i. e. the total number of receive antennas including all $K \mathrm{MSs}$ is given by $n_{\mathrm{R}}=\sum_{k=1}^{K} n_{\mathrm{R} k}$. The $\left(n_{\mathrm{R} k} \times 1\right)$ user specific symbol vector $\mathbf{c}_{k}$ to be transmitted by the BS is given by

$$
\mathbf{c}_{k}=\left(c_{k, 1}, c_{k, 2}, \ldots, c_{k, n_{\mathrm{R} k}}\right)^{\mathrm{T}} .
$$

The vector $\mathbf{c}_{k}$ is preprocessed before its transmission by multiplying it with the $\left(n_{\mathrm{T}} \times n_{\mathrm{R} k}\right)$ DL preprocessing matrix $\mathbf{R}_{k}$ and results in the $\left(n_{\mathrm{T}} \times 1\right)$ user-specific transmit vector

$$
\mathbf{s}_{k}=\mathbf{R}_{k} \mathbf{c}_{k} .
$$

After DL transmitter preprocessing, the $n_{\mathrm{T}^{-}}$ component signal $\mathbf{s}$ transmitted by the BS to the $K$ MSs results in

$$
\mathbf{s}=\sum_{k=1}^{K} \mathbf{s}_{k}=\mathbf{R} \mathbf{c}
$$

with the $\left(n_{\mathrm{T}} \times n_{\mathrm{R}}\right)$ preprocessing matrix

$$
\mathbf{R}=\left(\mathbf{R}_{1}, \mathbf{R}_{2}, \ldots, \mathbf{R}_{K}\right)
$$

In (3), the overall $\left(n_{\mathrm{R}} \times 1\right)$ transmitted DL data vector c combines all $K$ DL transmit vectors $\mathbf{c}_{k}$ (with $k=$ $1,2, \ldots, K)$ and is given by

$$
\mathbf{c}=\left(\mathbf{c}_{1}^{\mathrm{T}}, \mathbf{c}_{2}^{\mathrm{T}} \ldots, \mathbf{c}_{K}^{\mathrm{T}}\right)^{\mathrm{T}} .
$$

At the receiver side, the $\left(n_{\mathrm{R} k} \times 1\right)$ vector $\mathbf{u}_{k}$ of the $k$ th MS is given by

$$
\mathbf{u}_{k}=\mathbf{H}_{k} \mathbf{s}+\mathbf{n}_{k}=\mathbf{H}_{k} \mathbf{R} \mathbf{c}+\mathbf{n}_{k} .
$$

and can be expressed by

$$
\mathbf{u}_{k}=\mathbf{H}_{k} \mathbf{R}_{k} \mathbf{c}_{k}+\sum_{i=1, i \neq k}^{K} \mathbf{H}_{k} \mathbf{R}_{i} \mathbf{c}_{i}+\mathbf{n}_{k},
$$

where the MSs received signals experience both multi-user and multi-antenna interferences. In (6), the $\left(n_{\mathrm{R} k} \times n_{\mathrm{T}}\right)$ channel matrix $\mathbf{H}_{k}$ connects the $n_{\mathrm{T}} \mathrm{BS}$ specific transmit antennas with the $n_{\mathrm{R} k}$ receive antennas of the $k$ th MS.

It is quite common to assume that the coefficients of the $\left(n_{\mathrm{R} k} \times n_{\mathrm{T}}\right)$ channel matrix $\mathbf{H}_{k}$ are independent and Rayleigh distributed with equal variance. However, in many cases correlations between the transmit antennas as well as between the receive antennas can't be neglected. There are several methods to model and characterize antenna signal correlation into a MIMO channel model for Rayleigh flat-fading channels. In this work it is assumed that the correlation among receive antennas is independent of the correlation between transmit antennas. The way to include the antenna signal correlation into the MIMO channel model for Rayleigh flat-fading like channels is given by (Durgin and Rappaport, 1999; Zelst and Hammerschmidt, 2002) and results in

$$
\mathbf{H}_{k}=\mathbf{H}_{\mathrm{Rx}}^{1 / 2} \cdot \mathbf{G} \cdot \mathbf{H}_{\mathrm{Tx}}^{1 / 2},
$$

where $\mathbf{G}$ is a $\left(n_{\mathrm{R} k} \times n_{\mathrm{T}}\right)$ uncorrelated channel matrix with independent, identically distributed complex Gaussian zero-mean unit variance elements and where $(\cdot)^{1 / 2}$ stands for the square root of a matrix. The $\left(n_{\mathrm{R} k} \times n_{\mathrm{R} k}\right)$ matrix $\mathbf{H}_{\mathrm{Rx}}$ is used to model the correlation between the $k$ th MS receive antennas. Moreover, the $\left(n_{T} \times n_{T}\right)$ transmit correlation matrix $\mathbf{H}_{\mathrm{Tx}}$ models the correlation between the transmit antennas.

The interference, which is introduced by the channel matrix $\mathbf{H}_{k}$, requires appropriate signal processing strategies. A popular technique is based on the SVD of the system matrix $\mathbf{H}_{k}$ as described in (Ahrens and Benavente-Peces, 2010). Therein, after pre- and postprocessing of the transmitted and received signal vectors, the user-specific decision variables result in

$$
\mathbf{y}_{k}=\mathbf{V}_{k \mathrm{u}} \mathbf{P}_{k} \mathbf{c}_{k}+\mathbf{w}_{k},
$$




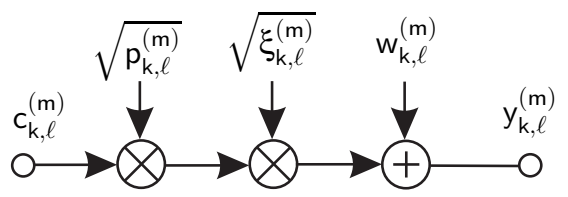

Figure 1: Resulting $k$ th user-specific system model per MIMO layer $\ell$ (with $\ell=1,2, \ldots, n_{\mathrm{R} k}$ ) and per transmitted symbol block $m$.

where interferences between the different antenna data streams as well as MUI (multi-user interference) imposed by the other users are avoided as shown in (Ahrens and Benavente-Peces, 2010). In (9), the $\left(n_{\mathrm{R} k} \times n_{\mathrm{R} k}\right)$ diagonal matrix $\mathbf{V}_{k \mathrm{u}}$ contains the nonzero square roots of the eigenvalues of $\mathbf{H}_{k}^{\mathrm{H}} \mathbf{H}_{k}$, e.g.,

$$
\mathbf{V}_{k \mathrm{u}}=\left[\begin{array}{cccc}
\sqrt{\xi_{k, 1}} & 0 & \cdots & 0 \\
0 & \sqrt{\xi_{k, 2}} & \ddots & \vdots \\
\vdots & \ddots & \ddots & \vdots \\
0 & 0 & \cdots & \sqrt{\xi_{k, n_{\mathrm{R} k}}}
\end{array}\right],
$$

and the user-specific $\left(n_{\mathrm{R} k} \times n_{\mathrm{R} k}\right)$ diagonal power allocation matrix is given by

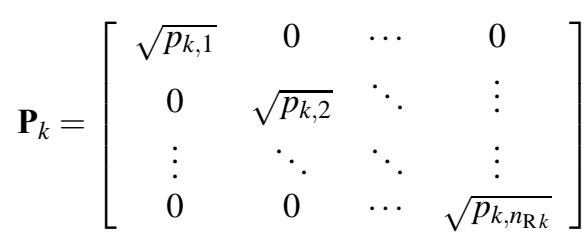

and simplifies to $\mathbf{P}_{k}=\sqrt{\beta} \mathbf{I}_{n_{\mathrm{R} k} \times n_{\mathrm{R} k}}$ with the parameter $\sqrt{\beta}$ taking the transmit-power constraint into account as highlighted in (Ahrens and Benavente-Peces, 2010). Finally the additive, white Gaussian noise (AWGN) vector is given by $\mathbf{w}_{k}$. The resulting system model is depicted in Fig. 1

\section{PERFORMANCE ANALYSIS}

In general, the user-specific quality of data transmission can be informally assessed by using the signalto-noise ratio (SNR) at the detector's input defined by the half vertical eye opening and the noise power per quadrature component according to

$$
\rho=\frac{(\text { Half vertical eye opening })^{2}}{\text { Noise Power }}=\frac{\left(U_{\mathrm{A}}\right)^{2}}{\left(U_{\mathrm{R}}\right)^{2}},
$$

which is often used as a quality parameter (Ahrens and Lange, 2008). The relationship between the signal-to-noise ratio $\rho=U_{\mathrm{A}}^{2} / U_{\mathrm{R}}^{2}$ and the bit-error probability evaluated for AWGN channels and $M$-ary
Quadrature Amplitude Modulation (QAM) is given by (Proakis, 2000)

$$
P_{\mathrm{BER}}=\frac{2}{\log _{2}(M)}\left(1-\frac{1}{\sqrt{M}}\right) \operatorname{erfc}\left(\sqrt{\frac{\rho}{2}}\right) .
$$

When applying the proposed system structure for the $k$ th user, the applied signal processing leads to different eye openings per activated MIMO layer $\ell$ (with $\ell=1,2, \ldots, L$ and $L \leq n_{\mathrm{R} k}$ describing the number of activated user-specific MIMO layers) and per transmitted symbol block $m$ according to

$$
U_{\mathrm{A} k}^{(\ell, m)}=\sqrt{p_{k, \ell}^{(m)}} \cdot \sqrt{\xi_{k, \ell}^{(m)}} \cdot U_{\mathrm{s} k}^{(\ell)},
$$

where $U_{\mathrm{s} k}^{(\ell)}$ denotes the half-level transmit amplitude assuming $M_{\ell}$-ary QAM, $\sqrt{\xi_{k, \ell}^{(m)}}$ represents the corresponding positive square roots of the eigenvalues of the matrix $\mathbf{H}_{k}^{\mathrm{H}} \mathbf{H}_{k}$ and $\sqrt{p_{k, \ell}^{(m)}}$ represents the corresponding power allocation weighting parameters. Together with the noise power per quadrature component, introduced by the additive, white Gaussian noise (AWGN) vector $\mathbf{w}_{k}$ in (9), the $k$ th user-specific SNR per MIMO layer $\ell$ at the time $m$ becomes

$$
\rho_{k}^{(\ell, m)}=\frac{\left(U_{\mathrm{A} k}^{(\ell, m)}\right)^{2}}{U_{\mathrm{R}}^{2}} .
$$

Using the parallel transmission over $L \leq n_{\mathrm{R} k}$ MIMO layers, the overall mean transmit power becomes $P_{\mathrm{s} k}=\sum_{\ell=1}^{L} P_{\mathrm{s} k}^{(\ell)}$. Considering QAM constellations, the average transmit power $P_{\mathrm{s} k}^{(\ell)}$ per MIMO layer $\ell$ may be expressed as (Proakis, 2000)

$$
P_{\mathrm{s} k}^{(\ell)}=\frac{2}{3}\left(U_{\mathrm{s} k}^{(\ell)}\right)^{2}\left(M_{k \ell}-1\right) .
$$

Combining (15) and (16) together with (14), the layerspecific SNR at the time $m$ results in

$$
\rho_{k}^{(\ell, m)}=p_{k, \ell}^{(m)} \xi_{k, \ell}^{(m)} \frac{3}{2\left(M_{k \ell}-1\right)} \frac{P_{\mathrm{s} k}^{(\ell)}}{U_{\mathrm{R}}^{2}} .
$$

Assuming that the transmit power is uniformly distributed over the number of activated MIMO layers, i. e., $P_{\mathrm{s} k}^{(\ell)}=P_{\mathrm{s} k} / L$, the layer-specific signal-to-noise ratio at the time $m$, defined in (17), results with the ratio of symbol energy to noise power spectral density $E_{\mathrm{s}} / N_{0}=P_{\mathrm{s} k} /\left(2 U_{\mathrm{R}}^{2}\right)$ in

$$
\rho_{k}^{(\ell, m)}=p_{k, \ell}^{(m)} \xi_{k, \ell}^{(m)} \frac{3}{L\left(M_{k \ell}-1\right)} \frac{E_{\mathrm{s}}}{N_{0}} .
$$

In order to transmit at a fixed data rate while maintaining the best possible integrity, i.e., bit-error rate, an appropriate number of user-specific MIMO layers 
Table 1: Investigated user-specific QAM transmission modes.

\begin{tabular}{ccccc}
\hline throughput & layer 1 & layer 2 & layer 3 & layer 4 \\
\hline $8 \mathrm{bit} / \mathrm{s} / \mathrm{Hz}$ & 256 & 0 & 0 & 0 \\
$\mathbf{8} \mathrm{bit} / \mathrm{s} / \mathbf{H z}$ & $\mathbf{6 4}$ & $\mathbf{4}$ & $\mathbf{0}$ & $\mathbf{0}$ \\
$\mathbf{8} \mathrm{bit} / \mathbf{s} / \mathbf{H z}$ & $\mathbf{1 6}$ & $\mathbf{1 6}$ & $\mathbf{0}$ & $\mathbf{0}$ \\
$\mathbf{8} \mathrm{bit} / \mathrm{s} / \mathbf{H z}$ & $\mathbf{1 6}$ & $\mathbf{4}$ & $\mathbf{4}$ & $\mathbf{0}$ \\
$8 \mathrm{bit} / \mathrm{s} / \mathrm{Hz}$ & 4 & 4 & 4 & 4 \\
\hline
\end{tabular}

has to be used, which depends on the specific transmission mode, as detailed in Table 1 for the exemplarily investigated two-user multiuser system $\left(n_{\mathrm{R} k}=4\right.$ (with $k=1,2), K=2, n_{\mathrm{R}}=n_{\mathrm{T}}=8$ ). In order to avoid any signalling overhead, fixed transmission modes are used in this contribution regardless of the channel quality (Ahrens and Lange, 2008).

However, the user-specific BER of the uncoded MIMO system is dominated by the specific layers having the lowest SNR's. As a remedy, a MIMOlayer transmit PA scheme is required for minimizing the overall BER under the constraint of a limited total MIMO transmit power. The proposed PA scheme scales the half-level transmit amplitude $U_{\mathrm{s} k}^{(\ell)}$ of the $\ell$ th MIMO layer by the factor $\sqrt{\tilde{p}_{k, \ell}^{(m)}}$. This results in a MIMO layer-specific transmit amplitude of $U_{\mathrm{s} k}^{(\ell)} \sqrt{\tilde{p}_{k, \ell}^{(m)}}$ for the QAM symbol of the transmit data vector transmitted at the time $m$ over the MIMO layer $\ell$. Together with the DL preprocessing design, the layer-specific power allocation parameter at the time $m$ results in:

$$
\sqrt{p_{k, \ell}^{(m)}}=\sqrt{\beta^{(m)}} \sqrt{\tilde{p}_{k, \ell}^{(m)}}
$$

A natural choice is to opt for a PA scheme, which results in an identical signal-to-noise ratio

$$
\rho_{\mathrm{PA} k}^{(\ell, m)}=\frac{\left(U_{\mathrm{PA} k}^{(\ell, m)}\right)^{2}}{U_{\mathrm{R}}^{2}}=\tilde{p}_{k, \ell}^{(m)} \frac{3 \xi_{k, \ell}^{(m)} \beta^{(m)}}{L\left(M_{k \ell}-1\right)} \frac{E_{\mathrm{s}}}{N_{0}}
$$

for all activated MIMO layers at the time $m$, i.e., in

$$
\rho_{\mathrm{PA} k}^{(\ell, m)}=\mathrm{constant} \quad \ell=1,2, \cdots, L .
$$

The power to be allocated to each activated MIMO layer at the time $m$ can be shown to be calculated as follows (Ahrens and Lange, 2008):

$$
\tilde{p}_{k, \ell}^{(m)}=\frac{\left(M_{k \ell}-1\right)}{\xi_{k, \ell}^{(m)}} \cdot \frac{L}{\sum_{v=1}^{L} \frac{\left(M_{k v}-1\right)}{\xi_{k, v}^{(m)}}}
$$

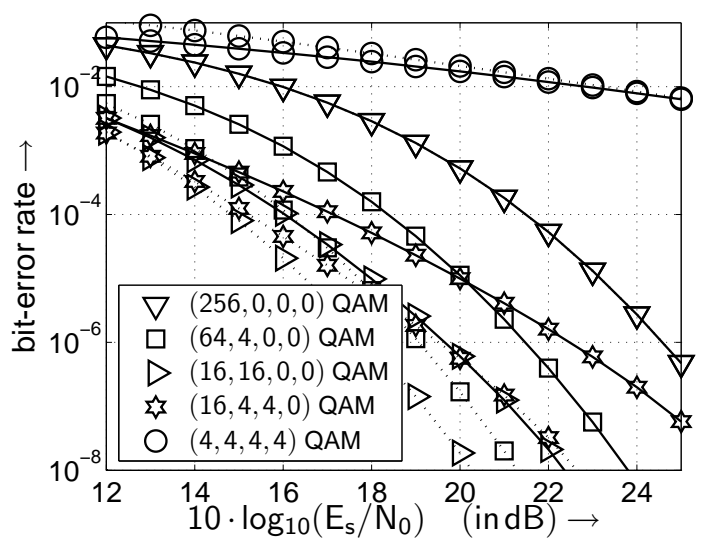

Figure 2: BER with PA (dotted line) and without PA (solid line) when using the transmission modes introduced in Tab. 1 and transmitting $8 \mathrm{bit} / \mathrm{s} / \mathrm{Hz}$ over uncorrelated frequency non-selective channels.

\section{RESULTS}

In this contribution fixed transmission modes are used regardless of the channel quality. Assuming predefined transmission modes, a fixed data rate can be guaranteed. Considering a frequency nonselective SDM (spatial division multiplexing) singleuser MIMO link $(K=1)$ composed of $n_{\mathrm{T}}=4$ transmit and $n_{\mathrm{R}}=4$ receive antennas, the obtained BER curves are depicted in Fig. 2 for the different QAM constellation sizes and MIMO configurations of Tab. 1, when transmitting at a bandwidth efficiency of 8 $\mathrm{bit} / \mathrm{s} / \mathrm{Hz}$. Assuming a uniform distribution of the transmit power over the number of activated MIMO layers, it turns out that not all MIMO layers have to be activated in order to achieve the best BERs.

PA can be used to balance the bit-error probabilities in the different number of activated MIMO layers. As shown in Fig. 2, unequal PA is only effective in conjunction with the optimum number of MIMO layers and at high SNR. Using all MIMO layers, our PA scheme would assign much of the total transmit power to the specific symbol positions per data block having the smallest singular values and hence the overall performance would deteriorate.

Analyzing an uncorrelated $(4 \times 4)$ MIMO channel, the corresponding distribution of the singularvalues is depicted in Fig. 3. Besides, assuming a transmit antenna separation of 10 -wavelength and a receive antenna separation of 4 -wavelength at a carrier frequency of $2.4 \mathrm{GHz}$, the resulting distribution of the singular-values within the correlated MIMO system is depicted in Fig. 4. Comparing the distribution of the singular-values depicted in Fig. 3 and 4 , the correlation shifts the pdf (probability density 


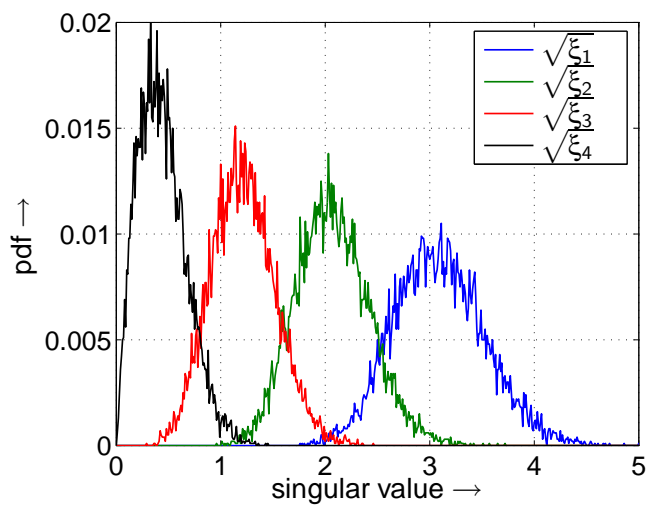

Figure 3: PDF (probability density function) of the layerspecific amplitudes $\sqrt{\xi_{\ell}}$ for uncorrelated frequency nonselective MIMO channels.

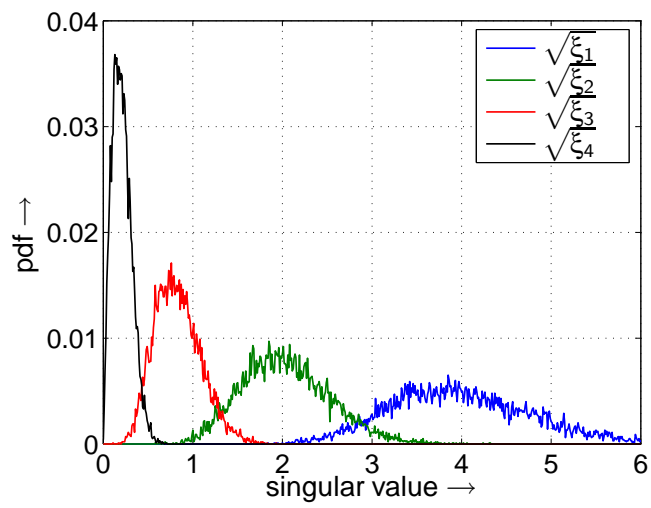

Figure 4: PDF (probability density function) of the layerspecific amplitudes $\sqrt{\xi_{\ell}}$ for correlated frequency nonselective MIMO channels.

function) of the largest singular-value to higher values at the cost of the remaining layers. Thus, taking the correlated MIMO channel instead of the uncorrelated one into consideration, we observe that the influence of the layer with the largest weighting factor increases. Since the performance of a MIMO transmission is strongly affected by the smallest singular values of the channel matrix, the statistical distribution of the smallest singular values is of great importance for the characterization of a MIMO transmission scheme. In consequence, as the ratio between the largest and smaller singular-value increases as the correlation between antennas increases, it is expected that the resulting BER increases with respect to the uncorrelated case. Thus, since the pdf dispersion of the singular values changes, the best uncoded solution for the uncorrelated MIMO channel doesn't necessarily lead to the best solution for the correlated one. The obtained BER curves are depicted in Fig. 5 for the different QAM constellation sizes and MIMO configura-

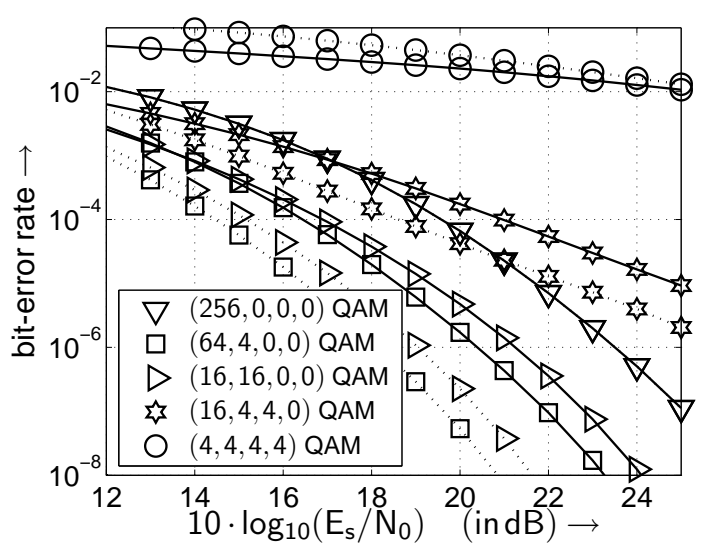

Figure 5: BER with PA (dotted line) and without PA (solid line) when using the transmission modes introduced in Tab. 1 and transmitting $8 \mathrm{bit} / \mathrm{s} / \mathrm{Hz}$ over correlated frequency non-selective channels.

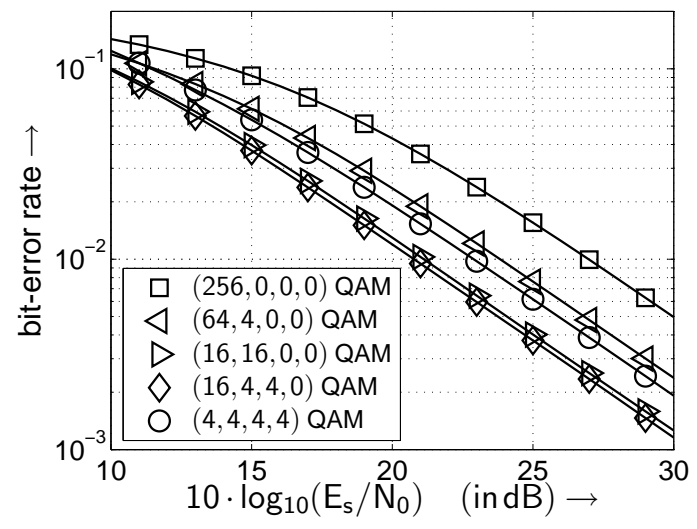

Figure 6: SVD-based user-specific BERs without PA when using the transmission modes introduced in Table 1 and transmitting $8 \mathrm{bit} / \mathrm{s} / \mathrm{Hz}$ over uncorrelated frequency nonselective channels.

tions of Tab. 1, when transmitting at a bandwidth efficiency of $8 \mathrm{bit} / \mathrm{s} / \mathrm{Hz}$. Here, the joint optimization of the number of activated MIMO layers along with the appropriate allocation of the transmit power allows us to minimize the overall BER under the constraint of a given fixed data throughput efficiently.

The parameters of the exemplarily studied twousers MIMO system are chosen as follows: $P_{\mathrm{s} k}=$ $1 \mathrm{~V}^{2}, n_{\mathrm{R} k}=4$ (with $k=1,2$ ), $K=2, n_{\mathrm{R}}=n_{\mathrm{T}}=$ 8. In this contribution a power with the dimension $(\text { voltage })^{2}$ (in $\mathrm{V}^{2}$ ) is used. At a real, constant resistor this value is proportional to the physical power (in $\mathrm{W})$. The obtained user-specific BER curves are depicted in Fig. 6 for the different QAM constellation sizes and MIMO configurations of Tab. 1 and confirm the obtained results within the single-user system $(K=1)$. Assuming a uniform distribution of the 


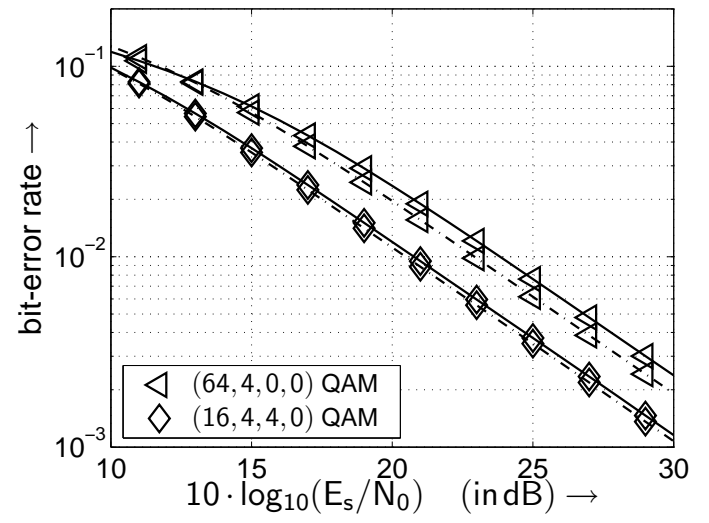

Figure 7: User-specific BERs with PA (dashed line) and without PA (solid line) when using the transmission modes introduced in Table 1 and transmitting $8 \mathrm{bit} / \mathrm{s} / \mathrm{Hz}$ over uncorrelated frequency non-selective channels.

transmit power over the number of activated MIMO layers, it still turns out that not all MIMO layers have to be activated in order to achieve the best BERs. PA can be used to balance the bit-error probabilities in the activated MIMO layers. The obtained BER curves are depicted in Fig. 7 and show, based on the chosen DL preprocessing design, only minor improvements by using adaptive PA within the investigated multiuser MIMO transmission scheme. Here, an equal power distribution seems to be a good choice.

\section{CONCLUSIONS}

Single- and multiuser MIMO systems in conjunction with SVD-assisted signal processing were investigated in this work. It turned out, that the choice of the number of bits per symbol as well as the number of activated MIMO layers substantially affects the performance of a MIMO system, suggesting that not all MIMO layers have to be activated in order to achieve the best BERs. The main goal was to find that specific combination of the QAM mode and the number of MIMO layers, which gives the best possible BER performance at a given fixed bit/s/Hz bandwidth efficiency. The $E_{\mathrm{s}} / N_{0}$ value required by each scheme at BER $10^{-2}$ was extracted from computer simulations and the best systems are shown in bold in Table 1. Moreover, it has been shown that the antenna correlation strongly affects the system performance. Here, the performed joint optimization of the number of activated MIMO layers along with the appropriate allocation of the transmit power allows us efficiently to minimize the overall BER under the constraint of a given fixed data throughput.

\section{REFERENCES}

Ahrens, A. and Benavente-Peces, C. (2010). ModulationMode and Power Assignment for SVD-assisted and Iteratively Detected Downlink Multiuser MIMO Systems. In International Conference on Wireless Information Networks and Systems (WINSYS), pages 107114, Athens (Greece).

Ahrens, A. and Lange, C. (2008). Modulation-Mode and Power Assignment in SVD-equalized MIMO Systems. Facta Universitatis (Series Electronics and Energetics), 21(2):167-181.

Choi, R. L. and Murch, R. D. (2003). New Transmit Schemes and Simplified Receivers for MIMO Wireless Communication Systems. IEEE Transactions on Wireless Communications, 2(6):1217-1230.

Choi, R. L. and Murch, R. D. (2004). A Transmit Preprocessing Technique for Multiuser MIMO Systems using a Decomposition Approach. IEEE Transactions on Wireless Communications, 3(1):20-24.

Durgin, G. D. and Rappaport, T. S. (1999). Effects of Multipath Angular Spread on the Spatial Cross-Correlation of Received Voltage Envelopes. In IEEE Vehicular Technology Conference (VTC), pages 996-1000, Houston, Texas, USA.

Joham, M., Utschick, W., and Nossek, J. A. (2005). Linear Transmit Processing in MIMO Communications Systems. IEEE Transactions on Signal Processing, 53(8):2700-2712.

Kühn, V. (2006). Wireless Communications over MIMO Channels - Applications to CDMA and Multiple Antenna Systems. Wiley, Chichester.

Liu, W., Yang, L. L., and Hanzo, L. (2008). SVD Assisted Joint Transmitter and Receiver Design for the Downlink of MIMO Systems. In IEEE 68th Vehicular Technology Conference (VTC), pages 1-5, Calgary.

Meurer, M., Baier, P. W., Weber, T., Lu, Y., and Papathanassiou, A. (2000). Joint Transmission: An Advantageous Downlink Concept for CDMA Mobile Radio Systems using Time Division Duplexing. Electronics Letters, 36(10):900-901.

Proakis, J. G. (2000). Digital Communications. McGrawHill, Boston.

Zelst, A. v. and Hammerschmidt, J. S. (2002). A Single Coefficient Spatial Correlation Model for Multiple-Input Multiple-Output (MIMO) Radio Channels. In 27th General Assembly of the International Union of Radio Science, Maastricht. 